Pacific

Journal of

Mathematics

\title{
SHOWING DISTINCTNESS OF SURFACE LINKS BY TAKING 2-DIMENSIONAL BRAIDS
}

\author{
INASA NAKAMURA
}




\title{
SHOWING DISTINCTNESS OF SURFACE LINKS BY TAKING 2-DIMENSIONAL BRAIDS
}

\author{
INASA NAKAMURA
}

\begin{abstract}
For an oriented surface link $S$, we can take a satellite construction called a 2-dimensional braid over $S$, which is a surface link in the form of a covering over $S$. We demonstrate that 2-dimensional braids over surface links are useful for showing the distinctness of surface links. We investigate nontrivial examples of surface links with free abelian groups of rank two, concluding that their link types are infinitely many.
\end{abstract}

\section{Introduction}

A surface link is the image of a smooth embedding of a closed surface into Euclidean space $\mathbb{R}^{4}$. Two surface links are equivalent if there is an orientation-preserving selfdiffeomorphism of $\mathbb{R}^{4}$ carrying one to the other. In this paper, we assume that surface links are oriented. In [Nakamura 2014a], we investigated a satellite construction called a 2-dimensional braid over an oriented surface link, and introduced its graphical presentation called an $m$-chart on a surface diagram. A 2-dimensional braid over a surface link $S$ is a surface link in the form of a covering over $S$, and can be regarded as an analog to a double of a classical link. One of the expected applications of the notion of a 2-dimensional braid is that it will provide us with a method for showing the distinctness of surface links. The aim of this paper is to demonstrate such use for 2-dimensional braids.

Our main theorem is as follows. For a positive integer $k$, let $\sigma_{1}, \sigma_{2}, \ldots, \sigma_{k}$ be the standard generators of the $(k+1)$-braid group. Take $X_{1}=\sigma_{1}^{2}$ and $X_{k}=$ $\sigma_{1}^{2} \sigma_{2} \sigma_{3} \cdots \sigma_{k}$, and let $\Delta$ be a $(k+1)$-braid with a positive half twist. Consider $S_{k}=\mathscr{Y}_{k+1}\left(X_{k}, \Delta^{2}\right)$, a torus-covering $T^{2}$-link determined from the $(k+1)$-braids $X_{k}$ and $\Delta^{2}$. We take the first component of $S_{k}$ to be the one determined from the first strand of $X_{k}$, and likewise the second component from the second strand; see Section 2 for the construction. Here, a $T^{2}$-link is a surface link each of whose components is of genus one.

MSC2010: primary 57Q45; secondary 57Q35, 57M25.

Keywords: surface link, 2-dimensional braid, chart, Roseman move, triple linking. 
Theorem 1.1. Abelian $T^{2}$-links of rank two, $S_{k}$ and $S_{l}$, are not equivalent for distinct positive integers $k$ and $l$. Thus, the link types of abelian $T^{2}$-links of rank two are infinitely many.

An abelian surface link of rank $n$ is a surface link whose link group is a free abelian group of rank $n$ [Ito and Nakamura 2014]; note that $n$ is the number of the components. We remark that our abelian $T^{2}$-links of rank two cannot be distinguished by using link groups, and that by a homological argument we cannot show that their link types are infinitely many, but only that there are two link types; see Section 2B. Our abelian $T^{2}$-link $S_{k}$ of rank two is a sublink of the surface link given in [Ito and Nakamura 2014], where we gave examples of abelian $T^{2}$-links of rank four, and we showed that their link types are infinitely many by calculations of triple linking numbers (see also Remark 2.3).

Triple linking numbers are integer-valued invariants of surface links with at least three components, so we cannot use them directly for our case. In order to overcome this situation, we take a 2-dimensional braid over $S_{k}$ such that each component of $S_{k}$ is split into two components. This has four components, so we can calculate triple linking numbers. A 2-dimensional braid over a surface link is obtained from the "standard" 2-dimensional braid by the addition of braiding information. Unfortunately, for the standard 2-dimensional braid, the triple linking number is trivial (Proposition 5.1). However, addition of braiding information makes a 2-dimensional braid with nontrivial triple linking, and enables us to show that $S_{k}$ and $S_{l}$ are not equivalent for distinct positive integers $k$ and $l$. For a similar result, we refer to Suciu's paper [1985] where it is shown that there are infinitely many ribbon 2-knots in $S^{4}$ with knot group the trefoil knot group.

The paper is organized as follows. In Section 2, we review torus-covering links and explain our example $S_{k}$, and we review triple linking numbers of torus-covering links. In Section 3, we discuss the notion of a 2-dimensional braid over a surface link. In Section 4, we observe that a 2-dimensional braid of degree $m$ over a surface link is presented by a finite graph called an $m$-chart on a surface diagram, and that 2-dimensional braids of degree $m$ are equivalent if their surface diagrams with $m$-charts are related by local moves called Roseman moves. In Section 5, we prove Proposition 5.1. In Section 6, we calculate triple linking numbers of a certain 2-dimensional braid over $S_{k}$ and prove Theorem 1.1.

\section{Abelian $T^{2}$-links of rank two}

The example $S_{k}$ given in Theorem 1.1 is a surface link called a torus-covering link. In this section, we review torus-covering $T^{2}$-links; see [Nakamura 2011] for details. We briefly observe that $S_{k}$ is an abelian surface link of rank two, and that we cannot show that the link types of our examples are infinitely many by using a 
homological argument. Further, we review a formula for the triple linking numbers of torus-covering links [Ito and Nakamura 2014].

2A. Torus-covering links. Let $T$ be a standard torus in $\mathbb{R}^{4}$, the boundary of an unknotted (standardly embedded) solid torus in $\mathbb{R}^{3} \times\{0\} \subset \mathbb{R}^{4}$.

Definition 2.1. A torus-covering $T^{2}$-link $S$ is a surface link in the form of a 2dimensional braid over the standard torus $T$, i.e., $S$ is a $T^{2}$-link in $\mathbb{R}^{4}$ such that $S$ is contained in a tubular neighborhood $N(T)$ and $\left.\pi\right|_{S}: S \rightarrow T$ is an unbranched covering map, where $\pi: N(T) \rightarrow T$ is the natural projection.

Let $S$ be a torus-covering $T^{2}$-link. Fix a base point $x_{0}=\left(x_{0}^{\prime}, x_{0}^{\prime \prime}\right)$ of $T=S^{1} \times S^{1}$. Take two simple closed curves on $T, \boldsymbol{m}=\partial B^{2} \times\left\{x_{0}^{\prime \prime}\right\}$ and $\boldsymbol{l}=\left\{x_{0}^{\prime}\right\} \times S^{1}$. Recall that $T$ is embedded as $T=\partial\left(B^{2} \times S^{1}\right) \subset \mathbb{R}^{3} \times\{0\} \subset \mathbb{R}^{4}$. Let us consider the intersections $S \cap \pi^{-1}(\boldsymbol{m}) \subset B^{2} \times \boldsymbol{m}$ and $S \cap \pi^{-1}(\boldsymbol{l}) \subset B^{2} \times \boldsymbol{l}$. They are regarded as closed $m$-braids in the 3-dimensional solid tori, where $m$ is the degree of the covering map $\left.\pi\right|_{S}: S \rightarrow T$. Cutting open the solid tori along the 2-disk $\pi^{-1}\left(x_{0}\right)=B^{2} \times\left\{x_{0}\right\}$, we obtain two $m$-braids $a$ and $b$. The assumption that $\left.\pi\right|_{S}$ is an unbranched covering implies that $a$ and $b$ commute. We call the commutative braids $(a, b)$ the basis braids of $S$. Conversely, starting from a pair of commutative $m$-braids $(a, b)$, we can uniquely construct a torus-covering $T^{2}$-link with basis braids $(a, b)$ [Nakamura 2011, Lemma 2.8]. For commutative $m$-braids $a$ and $b$, we denote by $\mathscr{S}_{m}(a, b)$ the torus-covering $T^{2}$-link with basis braids $(a, b)$.

2B. Our abelian $\boldsymbol{T}^{\mathbf{2}}$-links of rank two. We can verify that $S_{k}=\mathscr{Y}_{k+1}\left(X_{k}, \Delta^{2}\right)$ is an abelian surface link as follows. The link group of a torus-covering link $\mathscr{T}_{m}(a, b)$ is a quotient group of the classical link group of the closure of $a$ such that the abelianization is a free abelian group [Nakamura 2011, Proposition 3.1]. Since the link group of the closure of $X_{k}$, a Hopf link, is a free abelian group of rank two, so is the link group of $S_{k}$.

We remark that a homological argument cannot show that our examples are infinitely many, but only that there are two link types. Let us consider the one-point compactification of $\mathbb{R}^{4}$, and regard $S_{k}$ to be in the Euclidean 4-sphere $S^{4}$. Recall that we take the first and second components $F_{1}$ and $F_{2}$ of $S_{k}$ to be determined from the first and second strands of $X_{k}$, respectively. By Alexander's duality, we see that $H_{2}\left(S^{4}-F_{1} ; \mathbb{Z}\right) \cong H_{1}\left(F_{1} ; \mathbb{Z}\right)$, whence $\left[F_{2}\right]=\mu+k \lambda \in H_{2}\left(S^{4}-F_{1} ; \mathbb{Z}\right)$, where $(\mu, \lambda)$ is a preferred basis of $H_{1}\left(F_{1} ; \mathbb{Z}\right) \cong H_{2}\left(S^{4}-F_{1} ; \mathbb{Z}\right)$ represented by a meridian and a preferred longitude of $F_{1}$. Similarly, let us denote by $F_{1}^{\prime}$ and $F_{2}^{\prime}$ the first and second components of $S_{l}$. Then we can see that $\left[F_{2}^{\prime}\right]=\mu^{\prime}+l \lambda^{\prime} \in H_{2}\left(S^{4}-F_{1}^{\prime} ; \mathbb{Z}\right)$, where $\left(\mu^{\prime}, \lambda^{\prime}\right)$ is a preferred basis of $H_{1}\left(F_{1}^{\prime} ; \mathbb{Z}\right) \cong H_{2}\left(S^{4}-F_{1}^{\prime} ; \mathbb{Z}\right)$ represented by a meridian and a preferred longitude of $F_{1}^{\prime}$. Now, the standardly embedded tori $F_{1}$ and $F_{1}^{\prime}$ are related by an orientation-preserving self-diffeomorphism of $S^{4}$ if and 
only if

$$
\left(\begin{array}{l}
\mu^{\prime} \\
\lambda^{\prime}
\end{array}\right)=A\left(\begin{array}{l}
\mu \\
\lambda
\end{array}\right)
$$

for

$$
A=\left(\begin{array}{ll}
\alpha & \beta \\
\delta & \gamma
\end{array}\right) \in G L_{+}(2 ; \mathbb{Z})
$$

such that $\alpha+\beta+\gamma+\delta \equiv 0(\bmod 2)[$ Montesinos 1983], which implies that $\left[F_{2}\right]=\left[F_{2}^{\prime}\right] \in H_{2}\left(S^{4}-F_{1} ; \mathbb{Z}\right)$ if and only if $k \equiv l(\bmod 2)$ (see [Iwase 1988]).

Remark 2.2. The abelian surface link $S_{1}$, i.e., $\mathscr{Y}_{2}\left(\sigma_{1}^{2}, \sigma_{1}^{2}\right)$, is the twisted Hopf 2-link we will mention in the proof of Proposition 5.1; see also [Carter et al. 2001].

Remark 2.3. It is known [Kawauchi 1996, Theorem 6.3.1-Exercise 6.3.3] that for classical links, the rank of an abelian link is at most two, and for abelian links of rank two, there are exactly two link types: a positive and a negative Hopf link.

Remark 2.4. Set $T_{m}=\mathscr{Y}_{k+1}\left(X_{k}, X_{k}^{m}\right)$ for an integer $m$. It is known ([Boyle 1993], see also [Iwase 1988; Nakamura 2011]) that $T_{m}$ and $T_{n}$ are equivalent for $m \equiv n(\bmod 2)$. Fix the first component of $T_{m}$ in the form of the standard torus. By a homological argument as above, we see that $T_{m}$ cannot be taken to $T_{n}$ for $n \neq m$ by an orientation-preserving self-diffeomorphism of $\mathbb{R}^{4}$ relative to the first component.

2C. Triple linking numbers of torus-covering links. The triple linking number of a surface link $S$ is defined as follows [Carter et al. 2003, Definition 9.1]. For the $i$-th, $j$-th, and $k$-th components $F_{i}, F_{j}, F_{k}$ of $S$ with $i \neq j$ and $j \neq k$, the triple linking number $\mathrm{tlk}_{i, j, k}(S)$ of the $i$-th, $j$-th, and $k$-th components of $S$ is the total number of positive triple points minus the total number of negative triple points of a surface diagram of $S$ such that the top, middle, and bottom sheet are from $F_{i}, F_{j}$, and $F_{k}$, respectively. The triple linking number is a link bordism invariant [Carter et al. 2004; 2001; Sanderson 1987; 1993]; for other properties, see [Carter et al. 2003; 2004]. Triple linking numbers are useful for showing the distinctness of surface links with at least three components [Ito and Nakamura 2014; Nakamura 2012; 2014b].

From [Ito and Nakamura 2014], we have a formula for the triple linking numbers of a torus-covering $T^{2}$-link $\mathscr{S}_{m}(a, b)$. Let $A_{i}$ be the components of the closure of $a$ which are from the $i$-th component of $\mathscr{Y}_{m}(a, b)$. Take one of the connected components of $A_{i}$ and denote it by $A_{i}^{1}$. We define by the classical linking number

$$
\mathrm{lk}_{i, j}^{a}=\mathrm{lk}\left(A_{i}^{1}, A_{j}\right),
$$

where we regard $A_{i}^{1}$ and $A_{j}$ as oriented links in $\mathbb{R}^{3}$. The notation $\mathrm{lk}_{i, j}^{b}$ for the other basis braid is defined similarly. Note that $\mathrm{lk}_{i, j}^{a}$ does not depend on the choice of 
a connected component $A_{i}^{1}$ [Ito and Nakamura 2014, Remark 5.5], and note that $\mathrm{lk}_{i, j}^{a}$ is not always symmetric, i.e., $\mathrm{lk}_{i, j}^{a}$ is not always equal to $\mathrm{lk}_{j, i}^{a}$.

Now, for a torus-covering $T^{2}$-link, the triple linking number of the $i$-th, $j$-th, and $k$-th components is given by

$$
\operatorname{tlk}_{i, j, k}\left(\mathscr{S}_{m}(a, b)\right)=-\mathrm{lk}_{j, i}^{a} 1 \mathrm{k}_{j, k}^{b}+\mathrm{lk}_{j, k}^{a} \mathrm{lk}_{j, i}^{b},
$$

where $i \neq k$ and $j \neq k$ [Ito and Nakamura 2014, Theorem 5.4 and Remark 5.7].

\section{Two-dimensional braids over a surface link}

A 2-dimensional braid, also called a simple braided surface, over a 2-disk is analogous to a classical braid [Kamada 1992; 2002; Rudolph 1983]. We can modify this notion to a 2-dimensional braid over a closed surface [Nakamura 2011], and further to a 2-dimensional braid over a surface link [Carter et al. 2004, Section 2.4.2; Nakamura 2014a]. In this section, we review this notion of a 2-dimensional braid over a surface link.

3A. Two-dimensional braids over a surface link. We use 2-dimensional braids without branch points over a closed surface, so our definition here is restricted to such surfaces; see [Nakamura 2011; 2014a] for the general definition.

Let $\Sigma$ be a closed surface, let $B^{2}$ be a 2-disk, and let $m$ be a positive integer.

Definition 3.1. A closed surface $\widetilde{\Sigma}$ embedded in $B^{2} \times \Sigma$ is called a 2-dimensional braid over $\Sigma$ of degree $m$ if the restriction $\left.\pi\right|_{\tilde{\Sigma}}: \widetilde{\Sigma} \rightarrow \Sigma$ is an unbranched covering map of degree $m$, where $\pi: B^{2} \times \Sigma \rightarrow \Sigma$ is the natural projection.

Take a base point $x_{0}$ of $\Sigma$. Two 2-dimensional braids over $\Sigma$ of degree $m$ are equivalent if there is a fiber-preserving ambient isotopy of $B^{2} \times \Sigma$ rel $\pi^{-1}\left(x_{0}\right)$ which carries one to the other.

A surface link is said to be of type $\Sigma$ when it is the image of an embedding of $\Sigma$. Let $S$ be a surface link of type $\Sigma$, and let $N(S)$ be a tubular neighborhood of $S$ in $\mathbb{R}^{4}$.

Definition 3.2. A 2-dimensional braid $\tilde{S}$ over $S$ is the image of a 2-dimensional braid over $\Sigma$ in $B^{2} \times \Sigma$ by an embedding $B^{2} \times \Sigma \rightarrow \mathbb{R}^{4}$ which identifies $N(S)$ with $B^{2} \times \Sigma$ as a $B^{2}$-bundle over a surface. We define the degree of $\tilde{S}$ as that of $S$.

Two 2-dimensional braids $\widetilde{S}$ and $\widetilde{S}^{\prime}$ over surface links $S$ and $S^{\prime}$ are equivalent if there is an ambient isotopy of $\mathbb{R}^{4}$ carrying $\widetilde{S}$ to $\widetilde{S}^{\prime}$ and $N(S)=B^{2} \times S$ to $N\left(S^{\prime}\right)=B^{2} \times S^{\prime}$ as a $B^{2}$-bundle over a surface.

Equivalent 2-dimensional braids over surface links are also equivalent as surface links. A 2-dimensional braid $\widetilde{S}$ over $S$ is a specific satellite with companion $S$; see [Carter et al. 2004, Section 2.4.2] as well as [Lickorish 1997, Chapter 1]. 


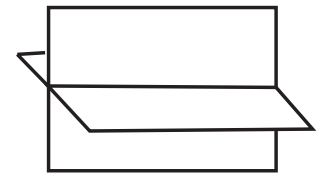

a double point curve

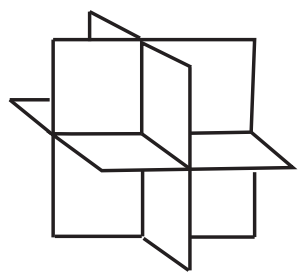

a triple point

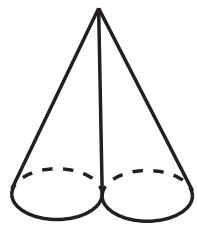

a branch point

Figure 1. The singularity of a surface diagram.

3B. Standard 2-dimensional braids. In this section, we define the standard 2dimensional braid over a surface link $S$. Using this notion, we will explain in the next section that a 2-dimensional braid is presented by a finite graph called an $m$-chart on a surface diagram $D$ of $S$. The standard 2-dimensional braid over $S$ is the 2-dimensional braid presented by an empty $m$-chart on $D$ [Nakamura 2014a].

We first review surface diagrams of a surface link $S$; see [Carter et al. 2004]. For a projection $p: \mathbb{R}^{4} \rightarrow \mathbb{R}^{3}$, the closure of the self-intersection set of $p(S)$ is called the singularity set. Let $p$ be a generic projection, meaning that the singularity set of the image $p(S)$ consists of double points, isolated triple points, and isolated branch points; see Figure 1. The closure of the singularity set forms a union of immersed arcs and loops, called double point curves. Triple points form the intersection points of the double point curves, and branch points form the end points. A surface diagram of $S$ is the image $p(S)$ equipped with over/under information along each double point curve with respect to the projection direction.

We define the $2 m$-braid $\tilde{\sigma}_{1}$ obtained from a 2 -braid $\sigma_{1}$ as follows. More generally, we construct an $m n$-braid $\tilde{b}$ from an $n$-braid $b$, needed for the proof of Theorem 1.1. Let $Q_{m}$ be $m$ interior points of $B^{2}$. For a standard generator $\sigma_{i}$ of an $n$-braid, let $\tilde{\sigma}_{i}$ be the $m n$-braid obtained from $\sigma_{i}$ in the form of a $Q_{m}$-bundle over $\sigma_{i}$ by splitting each strand into a bundle of $m$ parallel strands with a negative half twist at the initial points of each bundle; see Figure 2. The map taking $\sigma_{i}$ to $\tilde{\sigma}_{i}$ determines a homomorphism from the $n$-braid group to the $m n$-braid group. For an $n$-braid $b$, let $\tilde{b}$ denote the image of $b$ by this homomorphism.

Definition 3.3. Let $S$ be a surface link. A surface diagram $D$ of $S$ consists of the following local parts: around (1) a regular point, i.e., a nonsingular point,

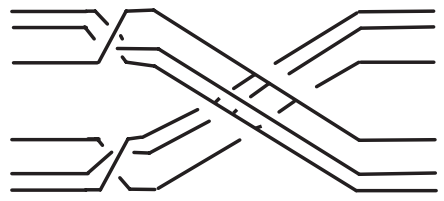

Figure 2. The $2 m$-braid $\widetilde{\sigma}_{1}$. 
(2) a double point curve, (3) a triple point, and (4) a branch point. The diagram around a regular point (1) consists of an embedded 2-disk $B^{2}$ with no singularities, and the diagram around a double-point curve (2) can be expressed as the product of a 2-braid $\sigma_{1}$ and an interval $I$.

We define the standard 2-dimensional braid over $S$ locally for such local parts of $D$ as follows: for (1), it is $m$ parallel copies of $B^{2}$, and for (2), it is the product of the $2 m$-braid $\widetilde{\sigma}_{1}$ and $I$. Then, for the other cases (3) and (4), the standard 2-dimensional braid is naturally defined [Nakamura 2014a, Definition 5.1 and Proposition 5.2].

\section{Chart presentation of 2-dimensional braids and Roseman moves}

In this section, we recall that a 2-dimensional braid of degree $m$ over a surface link $S$ is presented by a finite graph called an $m$-chart on a surface diagram $D$ of $S$. For two 2-dimensional braids of degree $m$, they are equivalent if their surface diagrams with $m$-charts are related by a finite sequence of local moves called Roseman moves. See [Nakamura 2014a].

4A. Chart presentation of 2-dimensional braids over a surface link. The graphical method called an $m$-chart on a 2-disk was introduced to present a simple surface braid which is a 2-dimensional braid over a 2-disk with trivial boundary condition [Kamada 1992; 2002]. By regarding an $m$-chart on a 2-disk as drawn on a 2 -sphere $S^{2}$, it presents a 2-dimensional braid over $S^{2}$ [Kamada 1992; 2002; Nakamura 2011]. This notion can be modified to an $m$-chart on a closed surface, and further to an $m$-chart on a surface diagram $D$ of a surface link $S$ [Nakamura 2011;2014a]. A 2-dimensional braid over $S$ is presented by an $m$-chart on $D$ [Nakamura 2014a].

In this paper, we treat 2-charts with vertices of degree 2 . We now review the graphical form of an $m$-chart of a 2-dimensional braid over a surface link. See [Nakamura 2014a] for details.

Let $\widetilde{S}$ be a 2-dimensional braid over a surface link $S$. Let $D$ be a surface diagram of $S$ by a projection $p: \mathbb{R}^{4} \rightarrow \mathbb{R}^{3}$ which is generic with respect to both $S$ and $\widetilde{S}$. We can assume that the singularity set of the surface diagram $p(\widetilde{S})$ is the union of the singularity set of the diagram of the standard 2-dimensional braid over $S$ and some finite graph $\Gamma$ [Nakamura 2014a, Theorem 5.5]. Project $\Gamma$ to $D$ by the projection $p(N(S))=B^{2} \times D \rightarrow D$. Thus we obtain a finite graph on the surface diagram $D$. An $m$-chart on a surface diagram $D$ is such a finite graph equipped with certain additional information of orientations and labels assigned to the edges, where $m$ is the degree of the 2-dimensional braid. Owing to the additional information, we can regain the original 2-dimensional braid from the $m$-chart on $D$ [Nakamura 2014a] (see also [Kamada 2002]).

We can define an $m$-chart on $D$ in graphical terms, where the labels of edges are from 1 to $m-1$; see [Nakamura 2014a, Definitions 5.3 and 5.4]. Around a double 


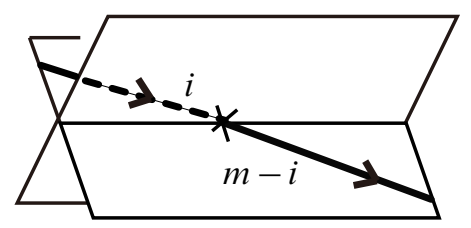

Figure 3. An $m$-chart around a double point curve. Here $i$ is one of $1, \ldots, m-1$. For simplicity, we omit the over/under information of each sheet.

point curve, an $m$-chart is as in Figure 3, with a vertex of degree 2. A 2-dimensional braid over $S$ is presented by an $m$-chart on $D$ [Nakamura 2014a, Theorem 5.5].

4B. Roseman moves. Roseman moves are local moves of surface diagrams, as illustrated in Figure 4. It is known [Roseman 1998] that two surface links are equivalent if and only if their surface diagrams are related by a finite sequence of Roseman moves and ambient isotopies of the diagrams in $\mathbb{R}^{3}$. In [Nakamura 2014a], we introduced the notion of Roseman moves for surface diagrams with $m$-charts.

An $m$-chart is said to be empty if it is an empty graph.

Definition 4.1. We define Roseman moves for surface diagrams with $m$-charts by the local moves as shown in Figures 4 and 5, where we regard the diagrams in Figure 4 as equipped with empty $m$-charts.

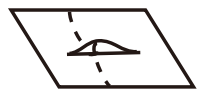

$\uparrow$
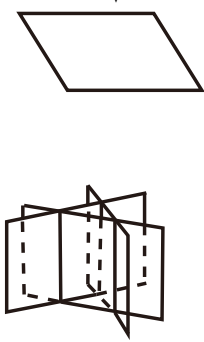

$\uparrow$

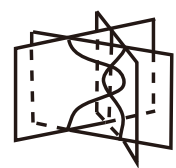

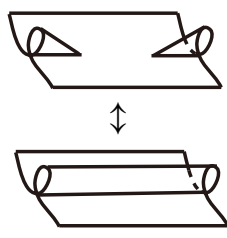

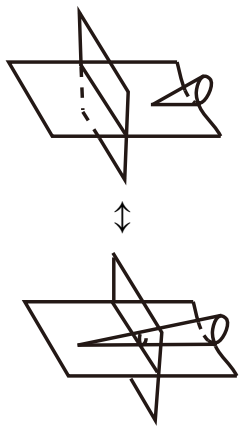

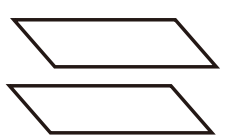

$\uparrow$
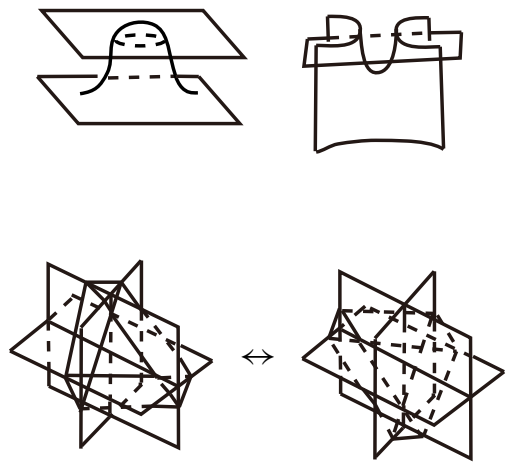

Figure 4. Roseman moves. We omit the over/under information of each sheet. 

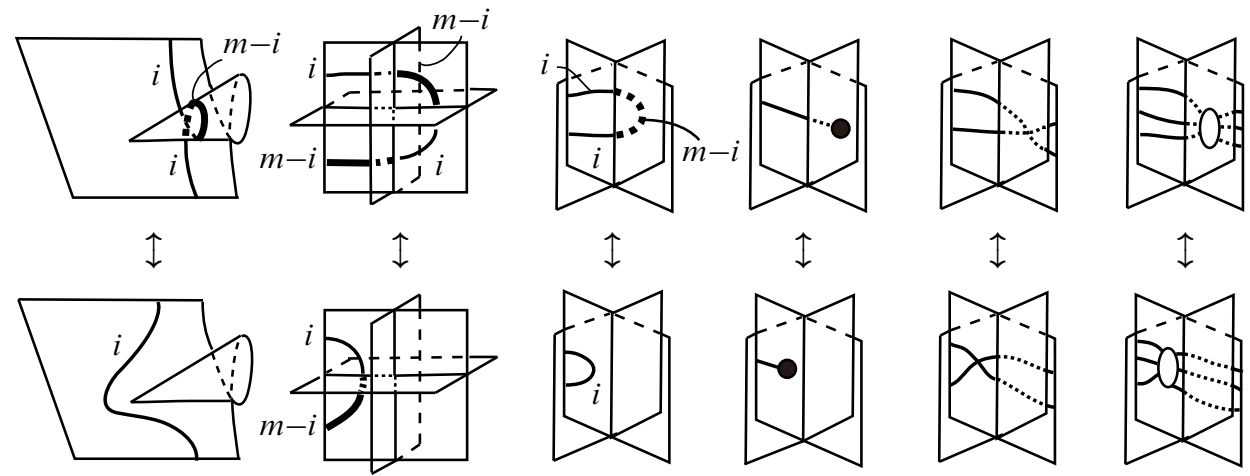

$\uparrow$

$\uparrow$

$\uparrow$

$\uparrow$
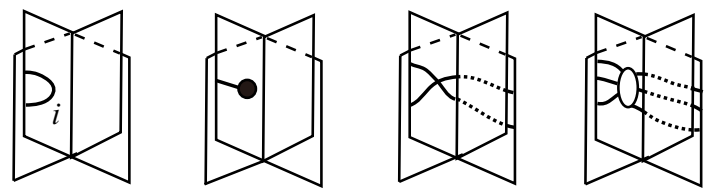

Figure 5. Roseman moves for surface diagrams with $m$-charts, where $i \in\{1, \ldots, m-1\}$. We omit the over/under information of each sheet, and orientations and labels of edges of $m$-charts.

Roseman moves for surface diagrams with $m$-charts, as illustrated in Figures 4 and 5, are well-defined. That is, for each pair of Roseman moves, the $m$-charts on the indicated diagrams present equivalent 2-dimensional braids [Nakamura 2014a, Theorem 6.2].

\section{Triple linking numbers of standard 2-dimensional braids}

Recall the triple linking numbers from Section 2C. We will say that a surface link $S$ has trivial triple linking if every triple linking number of $S$ is zero or $S$ consists of less than three components.

Proposition 5.1. For the standard 2-dimensional braid $\widetilde{S}$ over a surface link $S$, if $S$ has trivial triple linking, then so does $\widetilde{S}$.

Proof. Assume that $S$ has trivial triple linking. Recall from [Carter et al. 2001] that the link bordism class of a surface link is determined from triple linking numbers and double linking numbers (another kind of link bordism invariant), and that a surface link with trivial triple linking is link bordant to a split union of a finite number of trivial spheres and surface links called twisted Hopf 2-links, which has a surface diagram with no triple points (see also Remark 2.2). Hence, $S$ is link bordant to a surface link $S^{\prime}$ whose surface diagram has no triple points. By the well-definedness of Roseman moves, $\widetilde{S}$ is link bordant to the standard 2-dimensional braid $\widetilde{S^{\prime}}$ over $S^{\prime}$. Since the surface diagram of a standard 2-dimensional braid has triple points only around triple points of the companion surface [Nakamura 2014a], the surface diagram of $\widetilde{S}^{\prime}$ has no triple points. Thus $\tilde{S}$ is link bordant to a surface link with no triple points, which implies that $\widetilde{S}$ has trivial triple linking. 


\section{Proof of Theorem 1.1}

In this section, we consider a 2-dimensional braid $\widetilde{S}$ over a surface link $S$ presented by a 2 -chart consisting of a finite number of loops on a surface diagram of $S$. Here, a loop is a union of edges connected by vertices of degree 2 as in Figure 3. For our 2-charts, the edges are labeled by 1 and the orientations are coherent around a vertex of degree 2, so we can ignore the label information, and we regard the 2-chart on a surface diagram of $S$ as oriented loops. Further, we consider that the loops are on $S$ itself. By the well-definedness of Roseman moves, a 2-dimensional braid presented by a 2 -chart $\Gamma$ on $S$ is equivalent to the 2 -dimensional braid presented by a 2 -chart $f(\Gamma)$ on $f(S)$ for an orientation-preserving self-diffeomorphism $f$ of $\mathbb{R}^{4}$.

For a component $F$ of a torus-covering $T^{2}$-link, we take a preferred basis of $H_{1}(F ; \mathbb{Z})$ represented by a pair of simple closed curves $(\mu, \lambda)$ such that $\mu$ is a connected component of $F \cap \pi^{-1}(\boldsymbol{m})$, and $\lambda$ of $F \cap \pi^{-1}(\boldsymbol{l})$. Recall that $\pi: N(T) \rightarrow T$ is the natural projection for a standard torus $T$, and $\boldsymbol{m}$ and $\boldsymbol{l}$ are simple closed curves on $T$ given in Section 2A. We will use the same notation $(\mu, \lambda)$ for the preferred basis, and we call simple closed curves in the homology classes $\mu$ and $\lambda$ meridians and preferred longitudes of $F$, respectively. For a 2-chart $\Gamma$ on $F$ consisting of loops, we can assume that the intersections of the chart loops of $\Gamma$ with a meridian $\mu$ and a preferred longitude $\lambda$ of $F$ are transverse. We assign each intersection point the sign +1 or -1 according to whether it presents a positive or negative crossing, and we denote by $I(\mu, \Gamma)$ and $I(\lambda, \Gamma)$ the sum of the signs of the intersection points of $\Gamma$ with $\mu$ and $\lambda$. Note that we can assume that the chart loops are parallel by using local moves of charts called CI-moves of type (1) [Kamada 2002], and $I(\mu, \Gamma)$ and $I(\lambda, \Gamma)$ are well-defined for the homology classes $\mu$ and $\lambda$.

For the torus-covering $T^{2}$-link $S$, we take the first and second components of $S$ as those determined from the first and second strands of each basis braid of $S$, respectively. Similarly, for the 2-dimensional braid $\widetilde{S}$, we take the $i$-th component of $\widetilde{S}$ as the one determined from the $i$-th strand of each basis braid of $\widetilde{S}$ for $i=1,2,3,4$.

We first calculate the triple linking numbers of a 2-dimensional braid of degree 2 over $S_{k}$ as in Theorem 1.1.

Lemma 6.1. For the torus-covering $T^{2}$-link $S_{k}$ for a positive integer $k$, let us consider a 2-dimensional braid $\widetilde{S}_{k}$ of degree 2 over $S_{k}$, which is presented by a 2-chart $\Gamma$ consisting of loops on $S_{k}$ so that it consists of 4 components. Then $\operatorname{tlk}_{i, j, 3}\left(\widetilde{S}_{k}\right)=$ $\operatorname{tlk}_{i, j, 4}\left(\widetilde{S}_{k}\right)$ for $(i, j)=(1,2)$ or $(2,1)$, and $\operatorname{tlk}_{i, j, 1}\left(\widetilde{S}_{k}\right)=\operatorname{tlk}_{i, j, 2}\left(\widetilde{S}_{k}\right)$ for $(i, j)=$ $(3,4)$ or $(4,3)$.

Proof. The 2-dimensional braid $\widetilde{S}_{k}$ is also a torus-covering $T^{2}$-link. We denote by $(a, b)$ the basis braids presenting $\tilde{S}_{k}$. Since $\operatorname{lk}_{j, 3}^{c}=\mathrm{lk}_{j, 4}^{c}$ for $j=2,1$, and $\mathrm{lk}_{j, 1}^{c}=\mathrm{lk}_{j, 2}^{c}$ for $j=4,3(c=a, b)$, by (2-1) we have the result. 
Lemma 6.2. For the torus-covering $T^{2}$-link $S_{k}$, denote by $F_{1}$ and $F_{2}$ the first and second components of $S_{k}$. Let $\left(\mu_{i}, \lambda_{i}\right)$ be a preferred basis of $H_{1}\left(F_{i} ; \mathbb{Z}\right)$ for $i=1,2$. Consider a 2-dimensional braid $\widetilde{S}_{k}$, as in Lemma 6.1, such that $I\left(\mu_{i}, \Gamma\right)=2 p_{i}$ and $I\left(\lambda_{i}, \Gamma\right)=2 q_{i}$ for integers $p_{i}$ and $q_{i}$ for $i=1,2$. Then we have $\operatorname{tlk}_{1,2,3}\left(\widetilde{S}_{k}\right)=-k p_{1}+q_{1}$ and $\mathrm{tlk}_{2,3,4}\left(\widetilde{S}_{k}\right)=-p_{2}+q_{2}$.

Note that $\widetilde{S}_{k}$ consists of 4 components if and only if $I\left(\mu_{i}, \Gamma\right)$ and $I\left(\lambda_{i}, \Gamma\right)$ are even for $i=1,2$, since these conditions are equivalent to the condition that $\tilde{S}_{k} \cap \pi_{i}^{-1}(\mu)$ and $\widetilde{S}_{k} \cap \pi_{i}^{-1}(\lambda)$ are closed pure braids, where $\pi_{i}: N\left(F_{i}\right) \rightarrow F_{i}$ is the natural projection.

Proof. The 2-dimensional braid $\widetilde{S}_{k}$ is also a torus-covering $T^{2}$-link. We denote by $(a, b)$ the basis braids presenting $\widetilde{S}_{k}$. We use the notation given in Section 3B, taking $m=2$ and $n=k+1$. Then, $\mathrm{lk}_{2,1}^{a}$ is determined from the linking number coming from the linking consisting of $I\left(\mu_{1}, \Gamma\right)$ crossings and $\tilde{X}_{k}$. That is,

$$
\mathrm{lk}_{2,1}^{a}=p_{1}+\mathrm{lk}_{2,1}^{\tilde{X}_{k}}
$$

and similarly,

$$
\mathrm{lk}_{2,1}^{b}=q_{1}+\mathrm{lk}_{2,1}^{\widetilde{\Delta}^{2}} .
$$

By definition, for a braid $c$, the braid $\tilde{c}$ has a negative (respectively positive) half twist at the place which is a fiber of a point of each arc forming a positive (respectively negative) crossing of $c$. Hence,

$$
\begin{aligned}
& \mathrm{lk}_{2,1}^{\tilde{X}_{k}}=-\mathrm{lk}_{1,2}^{X_{k}} \\
& \mathrm{lk}_{2,1}^{\widetilde{\Delta}^{2}}=-\mathrm{lk}_{1,2}^{\Delta^{2}},
\end{aligned}
$$

from which it follows that

$$
\begin{aligned}
& \mathrm{lk}_{2,1}^{a}=p_{1}-\mathrm{lk}_{1,2}^{X_{k}} \\
& \mathrm{lk}_{2,1}^{b}=q_{1}-\mathrm{lk}_{1,2}^{\Delta^{2}} .
\end{aligned}
$$

Further, $\mathrm{lk}_{2,3}^{a}=\mathrm{lk}_{1,2}^{X_{k}}$ and $\mathrm{lk}_{2,3}^{b}=\mathrm{lk}_{1,2}^{\Delta^{2}}$. Thus $\mathrm{tlk}_{1,2,3}\left(\widetilde{S}_{k}\right)=-p_{1} \mathrm{lk}_{1,2}^{\Delta^{2}}+q_{1} \mathrm{lk}_{1,2}^{X_{k}}$ by (2-1). Since $\mathrm{lk}_{1,2}^{X_{k}}$ is the linking number of the closure of $X_{k}, 1 \mathrm{k}_{1,2}^{X_{k}}=1$. Since $F_{1}$ and $F_{2}$ are constructed by one strand and $k$ strands of $\Delta^{2}$, respectively, we have $\mathrm{lk}_{1,2}^{\Delta^{2}}=k$. Thus $\operatorname{tlk}_{1,2,3}\left(\widetilde{S}_{k}\right)=-k p_{1}+q_{1}$.

By the same argument, we have $\operatorname{llk}_{2,3,4}\left(\tilde{S}_{k}\right)=-p_{2} \mathrm{lk}_{2,1}^{\Delta^{2}}+q_{2} \mathrm{lk}_{2,1}^{X_{k}}$ by $(2-1)$, and $\operatorname{lk}_{2,1}^{X_{k}}=1$. Since $\Delta^{2}$ is a pure braid, we see that $\mathrm{k}_{2,1}^{\Delta^{2}}=1$. Thus tlk $\mathrm{k}_{2,3,4}\left(\widetilde{S}_{k}\right)=$ $-p_{2}+q_{2}$. 
Proof of Theorem 1.1. Let $k$ and $l$ be positive integers. We denote by $F_{1}$ and $F_{2}$ the first and second components of $S_{k}$, and by $F_{1}^{\prime}$ and $F_{2}^{\prime}$ the first and second components of $S_{l}$.

First we show that for $k \neq l$, there does not exist an orientation-preserving self-diffeomorphism of $\mathbb{R}^{4}$ carrying $F_{1}$ to $F_{1}^{\prime}$ and $F_{2}$ to $F_{2}^{\prime}$. Assume that there is such a diffeomorphism $f$. Let us consider a 2-dimensional braid over $S_{k}$, denoted by $\widetilde{S}_{k}^{1}$, which is presented by a 2 -chart $\Gamma$ on $S_{k}$ such that $\Gamma \cap F_{1}$ consists of loops with $I\left(\mu_{1}, \Gamma\right)=2 p$ and $I\left(\lambda_{1}, \Gamma\right)=2 q$, where $\left(\mu_{1}, \lambda_{1}\right)$ is a preferred basis of $H_{1}\left(F_{1} ; \mathbb{Z}\right)$, and $\Gamma \cap F_{2}=\varnothing$. Note that $\widetilde{S}_{k}^{1}$ consists of 4 components.

Since $f$ is an orientation-preserving diffeomorphism which carries $F_{1}$ to $F_{1}^{\prime}$, $\left.f\right|_{F_{1}}$ is an orientation-preserving diffeomorphism from a torus $F_{1}$ to a torus $F_{1}^{\prime}$. Let

$$
A=\left(\begin{array}{ll}
\alpha & \beta \\
\gamma & \delta
\end{array}\right) \in \mathrm{GL}_{+}(2, \mathbb{Z})
$$

be a matrix determined by

$$
\left(\begin{array}{l}
\mu_{1}^{\prime} \\
\lambda_{1}^{\prime}
\end{array}\right)=A\left(\begin{array}{l}
f_{*}\left(\mu_{1}\right) \\
f_{*}\left(\lambda_{1}\right)
\end{array}\right)
$$

where $\left(\mu_{1}^{\prime}, \lambda_{1}^{\prime}\right)$ is a preferred basis of $H_{1}\left(F_{1}^{\prime} ; \mathbb{Z}\right)$.

Put $\Gamma^{\prime}=f(\Gamma)$. By $f, \widetilde{S}_{k}^{1}$ is taken to a 2-dimensional braid $\widetilde{S}_{l}^{1}$ over $S_{l}$, presented by a 2 -chart $\Gamma^{\prime}$ on $S_{l}$ such that $\Gamma^{\prime} \cap F_{1}^{\prime}$ consists of loops and $\Gamma^{\prime} \cap F_{2}^{\prime}=\varnothing$. We see that $I\left(f_{*}\left(\mu_{1}\right), \Gamma^{\prime}\right)=I\left(\mu_{1}, \Gamma\right)=2 p$, and $I\left(f_{*}\left(\lambda_{1}\right), \Gamma^{\prime}\right)=I\left(\lambda_{1}, \Gamma\right)=2 q$. Set $p^{\prime}=I\left(\mu_{1}^{\prime}, \Gamma^{\prime}\right) / 2$ and $q^{\prime}=I\left(\lambda_{1}^{\prime}, \Gamma^{\prime}\right) / 2$; note that $p^{\prime}$ and $q^{\prime}$ are integers, since $\widetilde{S}_{l}^{1}$ consists of 4 components. It follows from (6-1) that

$$
\left(\begin{array}{l}
p^{\prime} \\
q^{\prime}
\end{array}\right)=A\left(\begin{array}{l}
p \\
q
\end{array}\right)
$$

Since the triple linking numbers $\mathrm{llk}_{1,2,3}$ for $\widetilde{S}_{k}^{1}$ and $\widetilde{S}_{l}^{1}$ are the same, Lemma 6.2 implies that

$$
-k p+q=-l p^{\prime}+q^{\prime} .
$$

Hence, it follows from (6-2) that $k p-q=(\alpha l-\gamma) p+(\beta l-\delta) q$. Since this equation holds true for any integers $p$ and $q$,

$$
\left(\begin{array}{c}
k \\
-1
\end{array}\right)=A^{T}\left(\begin{array}{c}
l \\
-1
\end{array}\right)
$$

where $A^{T}$ is the transposed matrix of $A$.

Next we consider another 2-dimensional braid over $S_{k}$, denoted by $\widetilde{S}_{k}^{2}$, presented by a 2 -chart $\widetilde{\Gamma}$ on $S_{k}$ such that $\widetilde{\Gamma} \cap F_{1}=\varnothing$ and $\widetilde{\Gamma} \cap F_{2}$ consists of loops on $F_{2}$, and 
moreover that $\tilde{\Gamma} \cap F_{2}$ is the preimage by the projection $N(T) \rightarrow T$ of a 2 -chart $\Gamma$ on the standard torus $T$ consisting of loops with $I(\boldsymbol{m}, \Gamma)=2 p$ and $I(\boldsymbol{l}, \Gamma)=2 q$, where $(\boldsymbol{m}, \boldsymbol{l})$ is a preferred basis of $T$. Note that $I\left(\mu_{2}, \widetilde{\Gamma}\right)=2 \mathrm{kp}$ and $I\left(\lambda_{2}, \widetilde{\Gamma}\right)=2 q$, where $\left(\mu_{2}, \lambda_{2}\right)$ is a preferred basis of $H_{1}\left(F_{2} ; \mathbb{Z}\right)$.

Let $g$ be an orientation-preserving diffeomorphism of $\mathbb{R}^{4}$ which carries $F_{2}$ sufficiently close to $F_{1}$ and $\left(\left.g\right|_{F_{i}}\right)_{*}=\mathrm{id}_{*}: H_{1}\left(F_{i} ; \mathbb{Z}\right) \rightarrow g_{*}\left(H_{1}\left(F_{i}\right) ; \mathbb{Z}\right)$ for $i=1,2$. Further, we assume that $T$ is sufficiently close to $F_{1}$. Then

$$
\left(\begin{array}{c}
\boldsymbol{m}^{\prime} \\
\boldsymbol{l}^{\prime}
\end{array}\right)=A\left(\begin{array}{c}
(f \circ g)_{*}(\boldsymbol{m}) \\
(f \circ g)_{*}(\boldsymbol{l})
\end{array}\right)
$$

where $\left(\boldsymbol{m}^{\prime}, \boldsymbol{l}^{\prime}\right)$ is a preferred basis of $T^{\prime}=(f \circ g)(T)$. Put $\Gamma^{\prime}=(f \circ g)(\Gamma)$. Then we have

$$
\left(\begin{array}{c}
I\left(\boldsymbol{m}^{\prime}, \Gamma^{\prime}\right) \\
I\left(\boldsymbol{l}^{\prime}, \Gamma^{\prime}\right)
\end{array}\right)=A\left(\begin{array}{c}
I(\boldsymbol{m}, \Gamma) \\
I(\boldsymbol{l}, \Gamma)
\end{array}\right)
$$

Put $S^{\prime}=(f \circ g)\left(S_{k}\right)$. The surface link $S^{\prime}$ is in the form of a 2-dimensional braid over $T^{\prime}$ of degree $k+1$. For the natural projection $\pi^{\prime}: N\left(T^{\prime}\right)=(f \circ g)(N(T)) \rightarrow$ $T^{\prime}$, a meridian $\boldsymbol{m}^{\prime}$, and a preferred longitude $\boldsymbol{l}^{\prime}$ of $T^{\prime}$, let us consider $S^{\prime} \cap \pi^{\prime-1}\left(\boldsymbol{m}^{\prime}\right)$ and $S^{\prime} \cap \pi^{\prime-1}\left(\boldsymbol{l}^{\prime}\right)$, which are closed $(k+1)$-braids in the 3-dimensional solid tori. In the same way as obtaining basis braids, we obtain $(k+1)$-braids from the closed braids by cutting open the solid tori along the 2-disk $\pi^{\prime-1}\left(x_{0}^{\prime}\right)$, where $x_{0}^{\prime}$ is the intersection point of $\boldsymbol{m}^{\prime}$ and $\boldsymbol{l}^{\prime}$. We denote the braids by $a$ and $b$. Note that here $T^{\prime}$ is a standard torus, and hence $(a, b)$ are basis braids, but we can apply the same argument if $T^{\prime}$ is not a standard torus. Since $S^{\prime}$ consists of two components, $a$ and $b$ satisfy one of the following three cases.

Case 1: The closure of $a$ is a link consisting of two components, and $b$ is a pure braid.

Case 2: Each of the closures of $a$ and $b$ is a link consisting of two components.

Case 3: The braid $a$ is a pure braid, and the closure of $b$ is a link consisting of two components.

Set $\tilde{\Gamma}^{\prime}=(\underset{\widetilde{\Gamma}}{f} \circ g)(\tilde{\Gamma})$. By $f \circ g, \widetilde{S}_{k}^{2}$ is taken to a 2-dimensional braid ${\widetilde{S^{\prime}}}^{\prime}$ presented by a 2 -chart $\widetilde{\Gamma}^{\prime}$ on $S^{\prime}$. We denote by $F^{\prime}$ the component $(f \circ g)\left(F_{2}\right)$ of $S^{\prime}$, and we denote by $\left(\mu^{\prime}, \lambda^{\prime}\right)$ a preferred basis of $H_{1}\left(F^{\prime} ; \mathbb{Z}\right)$. Since $\widetilde{\Gamma} \cap F_{2}$ is in the form of the preimage by $N(T) \rightarrow T$ of the 2-chart $\Gamma$ on $T, \widetilde{\Gamma}^{\prime} \cap F^{\prime}$ is in the form of the preimage by $N\left(T^{\prime}\right) \rightarrow T^{\prime}$ of the 2 -chart $\Gamma^{\prime}$ on $T^{\prime}$. Hence, $I\left(\mu^{\prime}, \tilde{\Gamma}^{\prime}\right)=i \cdot I\left(\boldsymbol{m}^{\prime}, \Gamma^{\prime}\right)$ 
and $I\left(\lambda^{\prime}, \widetilde{\Gamma}^{\prime}\right)=j \cdot I\left(\boldsymbol{l}^{\prime}, \Gamma^{\prime}\right)$ for $(i, j)=(k, 1)$ for Case $1,(k, k)$ for Case 2 , and $(1, k)$ for Case 3 . Thus

$$
\left(\begin{array}{c}
I\left(\mu^{\prime}, \widetilde{\Gamma}^{\prime}\right) \\
I\left(\lambda^{\prime}, \widetilde{\Gamma}^{\prime}\right)
\end{array}\right)=B\left(\begin{array}{c}
I\left(\boldsymbol{m}^{\prime}, \Gamma^{\prime}\right) \\
I\left(\boldsymbol{l}^{\prime}, \Gamma^{\prime}\right)
\end{array}\right)
$$

where $B$ is a diagonal matrix $\operatorname{diag}(i, j)$ such that $(i, j)=(k, 1)$ for Case $1,(k, k)$ for Case 2, and $(1, k)$ for Case 3.

Put $h=f \circ(f \circ g)^{-1}$. Then $h$ is an orientation-preserving self-diffeomorphism of $\mathbb{R}^{4}$ which carries $S^{\prime}$ to $S_{l}$. In particular, $h$ carries $F^{\prime}$ to the second component $F_{2}^{\prime}$ of $S_{l}$. Let

$$
C=\left(\begin{array}{ll}
\alpha^{\prime} & \beta^{\prime} \\
\gamma^{\prime} & \delta^{\prime}
\end{array}\right) \in \mathrm{GL}_{+}(2, \mathbb{Z})
$$

be a matrix determined by

$$
\left(\begin{array}{l}
\mu_{2}^{\prime} \\
\lambda_{2}^{\prime}
\end{array}\right)=C\left(\begin{array}{l}
h_{*}\left(\mu^{\prime}\right) \\
h_{*}\left(\lambda^{\prime}\right)
\end{array}\right)
$$

where $\left(\mu_{2}^{\prime}, \lambda_{2}^{\prime}\right)$ is a preferred basis of $H_{1}\left(F_{2}^{\prime} ; \mathbb{Z}\right)$. Put $\Gamma^{\prime \prime}=h\left(\widetilde{\Gamma}^{\prime}\right)$. Then

$$
\left(\begin{array}{l}
I\left(\mu_{2}^{\prime}, \Gamma^{\prime \prime}\right) \\
I\left(\lambda_{2}^{\prime}, \Gamma^{\prime \prime}\right)
\end{array}\right)=C\left(\begin{array}{c}
I\left(\mu^{\prime}, \widetilde{\Gamma}^{\prime}\right) \\
I\left(\lambda^{\prime}, \widetilde{\Gamma}^{\prime}\right)
\end{array}\right)
$$

Set $p^{\prime \prime}=I\left(\mu_{2}^{\prime}, \Gamma^{\prime \prime}\right) / 2$ and $q^{\prime \prime}=I\left(\lambda_{2}^{\prime}, \Gamma^{\prime \prime}\right) / 2$, which are both integers. Since $I(\boldsymbol{m}, \Gamma)=2 p$ and $I(\boldsymbol{l}, \Gamma)=2 q$, together with (6-5)-(6-7), we have

$$
\left(\begin{array}{l}
p^{\prime \prime} \\
q^{\prime \prime}
\end{array}\right)=(C B A)\left(\begin{array}{l}
p \\
q
\end{array}\right) \text {. }
$$

By the composite diffeomorphism $h \circ f \circ g=f, \widetilde{S}_{k}^{2}$ is taken to a 2-dimensional braid over $S_{l}$, which will be denoted by $\widetilde{S}_{l}^{2}$. Since tlk ${ }_{2,3,4}$ is the same for $\widetilde{S}_{k}^{2}$ and $\widetilde{S}_{l}^{2}$, together with $I\left(\mu_{2}, \widetilde{\Gamma}\right)=2 k p$ and $I\left(\lambda_{2}, \widetilde{\Gamma}\right)=2 q$, Lemma 6.2 implies that

$$
-k p+q=-p^{\prime \prime}+q^{\prime \prime}
$$

Since this equation holds true for any integers $p$ and $q$, it follows from (6-8) that

$$
\left(\begin{array}{c}
k \\
-1
\end{array}\right)=(C B A)^{T}\left(\begin{array}{c}
1 \\
-1
\end{array}\right) \text {. }
$$

Thus, together with (6-4),

$$
B^{T} C^{T}\left(\begin{array}{c}
1 \\
-1
\end{array}\right)=\left(\begin{array}{c}
l \\
-1
\end{array}\right),
$$


whence $i\left(\alpha^{\prime}-\gamma^{\prime}\right)=l$ and $j\left(\beta^{\prime}-\delta^{\prime}\right)=-1$. Let us assume $k>l>0$. For Cases 1 and $2, k\left(\alpha^{\prime}-\gamma^{\prime}\right)=l$ from the first equation. This contradicts the assumption that $k>l>0$. For Case 3, the second equation implies that $k\left(\delta^{\prime}-\beta^{\prime}\right)=1$, which contradicts the assumption that $k>1$. Thus, for $k \neq l$, there does not exist an orientation-preserving self-diffeomorphism of $R^{4}$ which carries $F_{1}$ to $F_{1}^{\prime}$ and $F_{2}$ to $F_{2}^{\prime}$.

Next we show that for $k \neq l$, there does not exist an orientation-preserving self-diffeomorphism of $R^{4}$ which carries $F_{1}$ to $F_{2}^{\prime}$ and $F_{2}$ to $F_{1}^{\prime}$. We discuss a similar argument as in the former case of a diffeomorphism which carries $F_{1}$ to $F_{1}^{\prime}$ and $F_{2}$ to $F_{2}^{\prime}$, using the same notation except where noted.

Assume that there is such a diffeomorphism $f$, and consider $\Gamma$ as before. Then, since $\mathrm{tlk}_{1,2,3}$ for $\widetilde{S}_{k}^{1}$ and $\mathrm{tlk}_{3,4,1}=\mathrm{tlk}_{4,3,2}$ (see Lemma 6.1) for $\widetilde{S}_{l}^{1}$ are the same, and since $\mathrm{tlk}_{4,3,2}=-\mathrm{tlk}_{2,3,4}$ [Carter et al. 2003], Lemma 6.2 implies that instead of (6-3) we have

$$
-k p+q=p^{\prime}-q^{\prime},
$$

where $p^{\prime}=I\left(\mu_{2}^{\prime}, \Gamma^{\prime}\right) / 2$ and $q^{\prime}=I\left(\lambda_{2}^{\prime}, \Gamma^{\prime}\right) / 2$. Hence instead of (6-4) we have

$$
\left(\begin{array}{c}
k \\
-1
\end{array}\right)=A^{T}\left(\begin{array}{c}
-1 \\
1
\end{array}\right) \text {. }
$$

Next we will consider another 2-dimensional braid $\widetilde{S}_{k}^{2}$ over $S_{k}$, presented by the 2-chart $\widetilde{\Gamma}$ as in the former case. Then, by the same argument, we have (6-8), where $p^{\prime \prime}=I\left(\mu_{1}^{\prime}, \Gamma^{\prime \prime}\right) / 2$ and $q^{\prime \prime}=I\left(\lambda_{1}^{\prime}, \Gamma^{\prime \prime}\right) / 2$.

By the composite diffeomorphism $h \circ f \circ g, \widetilde{S}_{k}^{2}$ is carried to a 2-dimensional braid over $S_{l}$, which will be denoted by $\widetilde{S}_{l}^{2}$. Since $\mathrm{tlk}_{2,3,4}$ for $\widetilde{S}_{k}^{2}$ and $\mathrm{tlk}_{3,1,2}=\mathrm{tlk}_{3,2,1}$ (see Lemma 6.1) for $\widetilde{S}_{l}^{2}$ are the same, and since $\mathrm{tlk}_{3,2,1}=-\mathrm{tlk}_{1,2,3}$ [Carter et al. 2003], together with $I\left(\mu_{2}, \widetilde{\Gamma}\right)=2 k p$ and $I\left(\lambda_{2}, \widetilde{\Gamma}\right)=2 q$, Lemma 6.2 implies that

$$
-k p+q=l p^{\prime \prime}-q^{\prime \prime} \text {. }
$$

Since this equation holds true for any integers $p$ and $q$, it follows from (6-8) that

$$
\left(\begin{array}{c}
k \\
-1
\end{array}\right)=(C B A)^{T}\left(\begin{array}{c}
-l \\
1
\end{array}\right) \text {. }
$$

Thus, together with (6-11),

$$
B^{T} C^{T}\left(\begin{array}{c}
-l \\
1
\end{array}\right)=\left(\begin{array}{c}
-1 \\
1
\end{array}\right),
$$

whence $i\left(-l \alpha^{\prime}+\gamma^{\prime}\right)=-1$ and $j\left(-l \beta^{\prime}+\delta^{\prime}\right)=1$. Let us assume $k>l>0$. Since at least one of $i$ and $j$ is $k$ for Cases 1,2 , and 3, these equations contradict the assumption that $k>1$. Thus, for $k \neq l$, there does not exist an orientation-preserving 
self-diffeomorphism of $R^{4}$ carrying $F_{1}$ to $F_{2}^{\prime}$ and $F_{2}$ to $F_{1}^{\prime}$. Thus $S_{k}$ and $S_{l}$ are not equivalent for positive integers $k \neq l$.

\section{Acknowledgments}

The author would like to thank Professors Seiichi Kamada, Shin Satoh, Hiroki Kodama, Takuya Sakasai, and the referees for their helpful comments. The author was supported by iBMath through the fund for Platform for Dynamic Approaches to Living Systems from MEXT.

\section{References}

[Boyle 1993] J. Boyle, “The turned torus knot in $S^{4}$ ", J. Knot Theory Ramifications 2:3 (1993), 239-249. MR 94i:57037 Zbl 0995.57502

[Carter et al. 2001] J. S. Carter, S. Kamada, M. Saito, and S. Satoh, "A theorem of Sanderson on link bordisms in dimension 4", Algebr. Geom. Topol. 1 (2001), 299-310. MR 2002d:57018 Zbl 0973.57010

[Carter et al. 2003] J. S. Carter, D. Jelsovsky, S. Kamada, L. Langford, and M. Saito, "Quandle cohomology and state-sum invariants of knotted curves and surfaces", Trans. Amer. Math. Soc. 355:10 (2003), 3947-3989. MR 2005b:57048 Zbl 1028.57003

[Carter et al. 2004] S. Carter, S. Kamada, and M. Saito, Surfaces in 4-space, Encyclopaedia of Mathematical Sciences 142, Springer, Berlin, 2004. MR 2005e:57065 Zbl 1078.57001

[Ito and Nakamura 2014] T. Ito and I. Nakamura, "On surface links whose link groups are abelian", Math. Proc. Cambridge Philos. Soc. 157:1 (2014), 63-77. MR 3211808 Zbl 1298.57018

[Iwase 1988] Z. Iwase, "Dehn-surgery along a torus $T^{2}$-knot”, Pacific J. Math. 133:2 (1988), 289-299. MR 89m:57020 Zbl 0629.57009

[Kamada 1992] S. Kamada, "Surfaces in $\mathbf{R}^{4}$ of braid index three are ribbon", J. Knot Theory Ramifications 1:2 (1992), 137-160. MR 93h:57039 Zbl 0763.57013

[Kamada 2002] S. Kamada, Braid and knot theory in dimension four, Mathematical Surveys and Monographs 95, American Mathematical Society, Providence, RI, 2002. MR 2003d:57050 Zbl 0993.57012

[Kawauchi 1996] A. Kawauchi, A survey of knot theory, Birkhäuser, Basel, 1996. MR 97k:57011 Zbl 0861.57001

[Lickorish 1997] W. B. R. Lickorish, An introduction to knot theory, Graduate Texts in Mathematics 175, Springer, New York, 1997. MR 98f:57015 Zbl 0886.57001

[Montesinos 1983] J. M. Montesinos, "On twins in the four-sphere. I", Quart. J. Math. Oxford Ser. (2) 34:134 (1983), 171-199. MR 86i:57025a Zbl 0522.57019

[Nakamura 2011] I. Nakamura, "Surface links which are coverings over the standard torus", Algebr. Geom. Topol. 11:3 (2011), 1497-1540. MR 2012g:57048 Zbl 1230.57022

[Nakamura 2012] I. Nakamura, "Triple linking numbers and triple point numbers of certain $T^{2}$-links", Topology Appl. 159:5 (2012), 1439-1447. MR 2879374 Zbl 1236.57030

[Nakamura 2014a] I. Nakamura, "Satellites of an oriented surface link and their local moves", Topology Appl. 164 (2014), 113-124. MR 3160466 Zbl 1295.57028

[Nakamura 2014b] I. Nakamura, "Surface links with free abelian groups", J. Math. Soc. Japan 66:1 (2014), 247-256. MR 3161400 Zbl 1297.57056 
[Roseman 1998] D. Roseman, "Reidemeister-type moves for surfaces in four-dimensional space", pp. 347-380 in Knot theory (Warsaw, 1995), edited by V. F. R. Jones et al., Banach Center Publ. 42, Polish Acad. Sci., Warsaw, 1998. MR 99f:57029 Zbl 0906.57010

[Rudolph 1983] L. Rudolph, "Braided surfaces and Seifert ribbons for closed braids", Comment. Math. Helv. 58:1 (1983), 1-37. MR 84j:57006 Zbl 0522.57017

[Sanderson 1987] B. J. Sanderson, "Bordism of links in codimension 2", J. London Math. Soc. (2) 35:2 (1987), 367-376. MR 88d:57023 Zbl 0622.57026

[Sanderson 1993] B. J. Sanderson, "Triple links in codimension 2", pp. 457-471 in Topology. Theory and applications, II (Pécs, 1989), edited by Á. Császár, Colloq. Math. Soc. János Bolyai 55, NorthHolland, Amsterdam, 1993. MR 96a:57056 Zbl 0815.57016

[Suciu 1985] A. I. Suciu, "Infinitely many ribbon knots with the same fundamental group", Math. Proc. Cambridge Philos. Soc. 98:3 (1985), 481-492. MR 87a:57025 Zbl 0596.57013

Received October 7, 2014. Revised March 27, 2015.

INASA NAKAMURA

Institute for Biology and Mathematics of Dynamic Cellular Processes

INTERDISCIPLINARY CENTER FOR MATHEMATICAL SCIENCES

GRADUATE SCHOOL OF MATHEMATICAL SCIENCES

THE UNIVERSITY OF TOKYO

3-8-1 Комава, Токуо 153-8914

JAPAN

inasa@ms.u-tokyo.ac.jp 


\title{
PACIFIC JOURNAL OF MATHEMATICS
}

\author{
msp.org/pjm
}

Founded in 1951 by E. F. Beckenbach (1906-1982) and F. Wolf (1904-1989)

\section{EDITORS}

Don Blasius (Managing Editor)

Department of Mathematics

University of California

Los Angeles, CA 90095-1555

blasius@math.ucla.edu

\author{
Paul Balmer \\ Department of Mathematics \\ University of California \\ Los Angeles, CA 90095-1555 \\ balmer@math.ucla.edu \\ Robert Finn \\ Department of Mathematics \\ Stanford University \\ Stanford, CA 94305-2125 \\ finn@math.stanford.edu \\ Sorin Popa \\ Department of Mathematics \\ University of California \\ Los Angeles, CA 90095-1555 \\ popa@math.ucla.edu
}

\author{
Vyjayanthi Chari \\ Department of Mathematics \\ University of California \\ Riverside, CA 92521-0135 \\ chari@math.ucr.edu \\ Kefeng Liu \\ Department of Mathematics \\ University of California \\ Los Angeles, CA 90095-1555 \\ liu@math.ucla.edu \\ Jie Qing \\ Department of Mathematics \\ University of California \\ Santa Cruz, CA 95064 \\ qing@ cats.ucsc.edu
}

\section{PRODUCTION}

Silvio Levy, Scientific Editor, production@msp.org

\section{SUPPORTING INSTITUTIONS}

ACADEMIA SINICA, TAIPEI

CALIFORNIA INST. OF TECHNOLOGY

INST. DE MATEMÁTICA PURA E APLICADA

KEIO UNIVERSITY

MATH. SCIENCES RESEARCH INSTITUTE

NEW MEXICO STATE UNIV.

OREGON STATE UNIV.

\author{
STANFORD UNIVERSITY \\ UNIV. OF BRITISH COLUMBIA \\ UNIV. OF CALIFORNIA, BERKELEY \\ UNIV. OF CALIFORNIA, DAVIS \\ UNIV. OF CALIFORNIA, LOS ANGELES \\ UNIV. OF CALIFORNIA, RIVERSIDE \\ UNIV. OF CALIFORNIA, SAN DIEGO \\ UNIV. OF CALIF., SANTA BARBARA
}

\author{
Daryl Cooper \\ Department of Mathematics \\ University of California \\ Santa Barbara, CA 93106-3080 \\ cooper@math.ucsb.edu \\ Jiang-Hua Lu \\ Department of Mathematics \\ The University of Hong Kong \\ Pokfulam Rd., Hong Kong \\ jhlu@maths.hku.hk \\ Paul Yang \\ Department of Mathematics \\ Princeton University \\ Princeton NJ 08544-1000 \\ yang@math.princeton.edu
}

These supporting institutions contribute to the cost of publication of this Journal, but they are not owners or publishers and have no responsibility for its contents or policies.

See inside back cover or msp.org/pjm for submission instructions.

The subscription price for 2015 is US \$420/year for the electronic version, and \$570/year for print and electronic.

Subscriptions, requests for back issues and changes of subscribers address should be sent to Pacific Journal of Mathematics, P.O. Box 4163, Berkeley, CA 94704-0163, U.S.A. The Pacific Journal of Mathematics is indexed by Mathematical Reviews, Zentralblatt MATH, PASCAL CNRS Index, Referativnyi Zhurnal, Current Mathematical Publications and Web of Knowledge (Science Citation Index).

The Pacific Journal of Mathematics (ISSN 0030-8730) at the University of California, c/o Department of Mathematics, 798 Evans Hall \#3840, Berkeley, CA 94720-3840, is published twelve times a year. Periodical rate postage paid at Berkeley, CA 94704, and additional mailing offices. POSTMASTER: send address changes to Pacific Journal of Mathematics, P.O. Box 4163, Berkeley, CA 94704-0163.

PJM peer review and production are managed by EditFLOW ${ }^{\circledR}$ from Mathematical Sciences Publishers.

\section{PUBLISHED BY}

\section{mathematical sciences publishers \\ nonprofit scientific publishing}

http://msp.org/

(C) 2015 Mathematical Sciences Publishers 


\section{PACIFIC JOURNAL OF MATHEMATICS}

Volume $278 \quad$ No. $1 \quad$ November 2015

Growth tight actions

Goulnara N. Arzhantseva, Christopher H. Cashen and JING TAO

A flag structure on a cusped hyperbolic 3-manifold

ELISHA FALBEL and RAFAEL SANTOS THEBALDI

A new upper bound for the Dirac operators on hypersurfaces

Nicolas GinouX, GeORGES Habib and Simon RaUlot

Games and elementary equivalence of $\mathrm{II}_{1}$-factors

ISAAC GOLDBRING and THOMAS SINCLAIR

Grossberg-Karshon twisted cubes and hesitant walk avoidance

MEGUMI HARADA and EUNJEONG LEE

Gamma factors of distinguished representations of $\mathrm{GL}_{n}(\mathbb{C})$

ALEXANDER KEMARSKY

The $W$-entropy formula for the Witten Laplacian on manifolds with 173 time dependent metrics and potentials

SONGZI LI and XIANG-DONG LI

A diagrammatic categorification of the affine $q$-Schur algebra $\hat{\boldsymbol{S}}(n, n) 201$ for $n \geq 3$

MARCO MACKAAY and ANNE-LAURE THIEL

Showing distinctness of surface links by taking 2-dimensional braids

INASA NAKAMURA

Correction to Modular $L$-values of cubic level 